\title{
Multi-objective optimization of high-sulfur natural gas purification plant
}

\author{
Jian-Feng Shang ${ }^{1,3} \cdot$ Zhong-Li Ji $^{1,2} \cdot$ Min Qiu ${ }^{1,2} \cdot$ Li-Min Ma ${ }^{1,2}$
}

Received: 25 June 2019 / Published online: 9 November 2019

(c) The Author(s) 2019

\begin{abstract}
There exists large space to save energy of high-sulfur natural gas purification process. The multi-objective optimization problem has been investigated to effectively reduce the total comprehensive energy consumption and further improve the production rate of purified gas. A steady-state simulation model of high-sulfur natural gas purification process has been set up by using ProMax. Seven key operating parameters of the purification process have been determined based on the analysis of comprehensive energy consumption distribution. To solve the problem that the process model does not converge in some conditions, back-propagation (BP) neural network has been applied to substitute the simulation model to predict the relative parameters in the optimization model. The uniform design method and the table $\mathrm{U}_{21}\left(10^{7}\right)$ have been applied to design the experiment points for training and testing BP model. High prediction accuracy can be achieved by using the BP model. Nondominated sorting genetic algorithm-II has been developed to optimize the two objectives, and 100 Pareto optimal solutions have been obtained. Three optimal points have been selected and evaluated further. The results demonstrate that the total comprehensive energy consumption is reduced by $13.4 \%$ and the production rate of purified gas is improved by $0.2 \%$ under the optimized operating conditions.
\end{abstract}

Keywords High-sulfur natural gas purification plant · Multi-objective optimization · Process simulation model · Thermodynamic analysis $\cdot$ BP neural network $\cdot$ Genetic algorithm

\section{Introduction}

With the rapid development of natural gas industry, consumption of natural gas has increased with an average annual growth rate 16\% in China from 2005 to 2015 (National Energy Administration 2016). Produced gas of sour gas field accounts for near $60 \%$ of China's natural gas production (Guo 2004). The major acid gases are hydrogen sulfide $\left(\mathrm{H}_{2} \mathrm{~S}\right)$ and carbon dioxide $\left(\mathrm{CO}_{2}\right)$. In the southwest of China, there exist a great number of gas fields with acid species,

\section{Edited by Xiu-Qiu Peng}

Li-Min Ma

malimin@cup.edu.cn

1 College of Mechanical and Transportation Engineering, China University of Petroleum, Beijing 102249, China

2 Beijing Key Laboratory of Process Fluid Filtration and Separation, Beijing 102249, China

3 Natural Gas Processing Plant of Sinopec Zhongyuan Oilfield Branch, Puyang 457061, China especially high $\mathrm{H}_{2} \mathrm{~S}$, in produced natural gas (Guo 2004). High concentration of acid species makes the purification process more complex and moreover leads to a substantial increase of total comprehensive energy consumption (TCEC) and operating costs compared with the conventional purification process (Li et al. 2007; Long et al. 2007; Jia et al. 2009). Figure 1 shows the TCEC of seven natural gas purifications with different sulfur contents in China (Azizkhani et al. 2014). The abbreviations of QX, DJ, ZX, DZ, LJZ, TSP and PG represent Quxian, Dianjiang, Zhongxian, Dazhu, Luojiazhai, Tieshanpo and Puguang, respectively, in Fig. 1. According to statistics, the TCEC of high-sulfur natural gas purification accounts for near 30\% of TCEC of the natural gas process in China and there is large space to reduce the TCEC of purification process ( $\mathrm{Li} 2012$ ).

The high-sulfur natural gas purification plant is mainly composed of five units, including the gas sweetening unit (GSU), the dehydration unit (DU), the sulfur recovery unit (SRU), the tail gas treatment unit (TGTU) and the acid water stripping unit (AWSU). In the natural gas purification system, there are a great number of devices and relative couples 


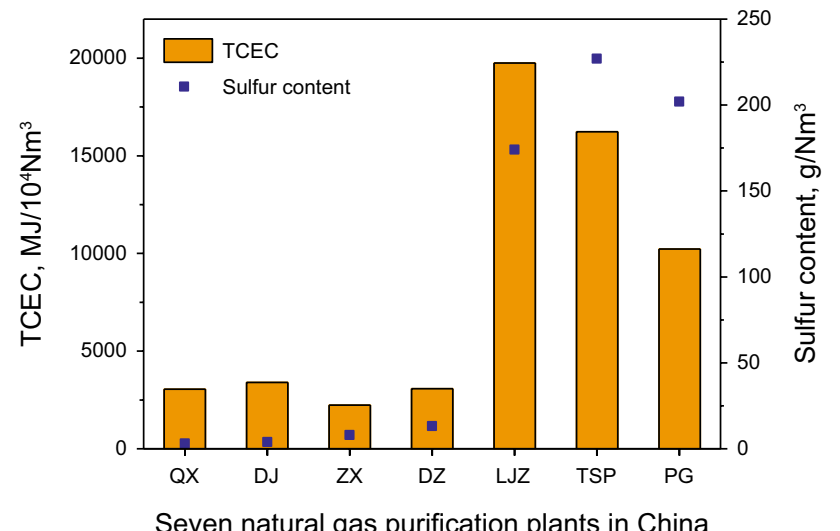

Fig. 1 TCEC of seven natural gas purification plants with different sulfur content

of operating parameters. The relation of dozens of operating parameters to TCEC is nonlinear and the coupling correlations between some parameters are strong, which makes the optimization problem of TCEC complicated and difficult to be solved by using the traditional optimization method ( $\mathrm{Yu}$ et al. 1998; Abdulrahman and Sebastine 2013).

In recent years, very few published articles research on the global optimization of operating conditions to reduce TCEC for the whole high-sulfur gas purification plant. Most optimization studies focus on the subsections, such as GSU (Jassim 2016; Peter et al. 2011; Qiu et al. 2014; Behroozsarand and Zamaniyan 2011; Al-Lagtah et al. 2015), DU (Santos et al. 2017; Rouzbahani et al. 2014), SRU (Manenti et al. 2014; Zarei et al. 2016; Adewale et al. 2016) and TGTU (Wahedi et al. 2015). A typical Iranian gas sweetening plant was simulated by HYSYS v3.1 and optimized by NSGA-II method (Behroozsarand and Zamaniyan 2011). Three objectives were minimizing of plant energy consumption, amine circulation rate and maximization of carbon dioxide recovery, respectively. Lekhwair natural gas sweetening plant was simulated by HYSYS v7.3 (Al-Lagtah et al. 2015). The main operating parameters, lean amine circulation rate, temperature and concentration, were optimized based on sensitivity analysis and keeping the device at its best operating range. Moreover, two modifications (conventional split-loop and modified split-loop) are simulated and discussed to meet the required gas purity at lower energy costs. A natural gas DU was simulated by a steady-state flow-sheet simulator, and a sensitivity analysis was performed based on the simulation results (Rouzbahani et al. 2014). For the process optimization, dry gas dew point was assumed as key factor and it was shown that a $10 \%$ increase in solvent molar flow rate is applicable in order to reduce dry gas dew point up to $6 \%$ without a significant rise in the total energy consumption and volatile organic compounds emission. An optimization of SRU was performed based on the multi-scale model and specific algorithms of BzzMath library (Manenti et al. 2014). The optimization problem has three input variables, furnace pressure, acid gas/air ratio and water temperature of waste heat boiler. The objective function is to maximize the production of sulfur and medium-pressure and low-pressure steam. The optimization result shows steam generation can be improved of more than $6 \%$ by preserving the amount of recovered sulfur. An optimization of SRU was carried out to maximize sulfur production and minimize COS emission while maintaining flow ratio $\mathrm{H}_{2} \mathrm{~S}$ to $\mathrm{SO}_{2}$ at 2 , in which the GA method was used. Three variables in the optimization function are the ratio of air to acid gas flow, the reaction furnace feed temperature and the steam pressure of waste heat boiler (Zarei et al. 2016). It was assessed that the economics of a TGTU rely on a temperature swing adsorption module. The optimization of minimum of net present worth costs (NPWC) of total capital investment, operating and bed replacement costs ensued during a 30-year project life was carried out (Wahedi et al. 2015).

Artificial neural networks have been widely applied to tackle complex issues. Since inception, they have been used in different engineering applications including prediction of key output parameters in the natural gas sweetening and dehydration process (Salooki et al. 2011; Ghiasi et al. 2014; Ahmadi et al. 2014; Darwish and Hilal 2008).

Biology inspired algorithms, which belong to intelligent numerical method, are generally divided into evolutionbased algorithm and swarm-based algorithm (Neri and Carlos 2012). Evolution-based algorithms, i.e., evolutionary algorithms, have strong adaptability and self-organization, including genetic algorithm (GA), evolutionary programming (EP), evolutionary strategy (ES), differential evolution (DE) algorithm, etc. (Cui et al. 2017). GA not only has simple, general and strong stability characteristics, but also has the parallel and global search ability, which makes it competent to solve the large-scale and nonlinear optimization problem (Louis et al. 2009; Dai et al. 2000; Azizkhani et al. 2014). Non-dominated sorting genetic algorithm-II (NSGA-II) was first proposed by Deb et al. (2000) based on the NSGA (Srinivas and Deb 1994). In recent years, NSGA-II has been applied in many engineering application to solve the multi-objective problems (Behroozsarand and Zamaniyan 2011; Damavandi et al. 2017; Singh and Das 2016; Deng et al. 2017; Boyaghchi and Chavoshi 2017). An simulation-optimization method based on the NSGA-II for the life cycle optimization of a novel process design for a more cost-effective, greener process for making chemicals from shale gas and bio-ethanol was developed (He and You 2015). New process integration approaches, graphical pinch technique, were proposed to optimize the process of unconventional gas field (Foo et al. 2016).

As mentioned above, there are few papers focused on the global optimization of the whole high-sulfur gas purification 
plant. In this paper, the objective is to explore an effective method for global optimization of the high-sulfur natural gas purification plants to reduce the TCEC and improve the purified gas production rate (PGPR) of it. Key operating parameters of the natural gas purification plant have been selected on the parametric sensitivity analysis and the energy consumption analysis for each unit. A steady-state simulation model of the high-sulfur natural gas purification process has been set up by using software ProMax to carry out this research. To solve the problem that the process simulation model does not converge in some operating conditions for the existence of nested loops in it, which leads to fail to supply the data required for further optimization, BP neural network has been applied to substitute the simulation model to predict the relative parameters in the global optimization model. The optimization model has been set up based on NSGA-II, and the Pareto optimal results have been achieved. In this work, the prototype natural gas process plant is located in the southwest of China.

\section{Process description and simulation}

\subsection{Process flow of high-sulfur natural gas purification plant}

The natural gas purification plant is mainly composed of five units, and the flow sheet of gas purification process is shown in Fig. 2. In the GSU, MDEA (methyldiethanolamine) is selected as the absorbent, and the feed gas is sent into the first absorption column (Abs-101), the reactor of COS hydrolysis (R-101) and the second absorption column (Abs102) successively. The rich MDEA solution will be regenerated in the regenerating column (Reg-104). After removal of the acid gas, the natural gas is sent to the DU, in which TEG (triethylene glycol) is selected as the absorbent. The natural gas is dehydrated in the absorption column (Abs201), and the rich TEG solution will be regenerated in the regenerating column (Reg-202). Flowing out of the GSU, the acid gas is fed into the SRU, which is mainly composed of the combustion reaction and the two-stage catalytic conversion reaction processes. In the combustion reaction furnace (F-301), nearly one-third $\mathrm{H}_{2} \mathrm{~S}$ of the acid gas is converted into $\mathrm{SO}_{2}$ and then the generated $\mathrm{SO}_{2}$ reacts with the left two-thirds $\mathrm{H}_{2} \mathrm{~S}$ to produce sulfur. And the high-temperature reaction heat is recovered by the waste heat boilers (first WHB and second WHB). The generated sulfur is condensed in the sulfur condenser (E-303/305/307) and sent into the liquid sulfur pool. To improve sulfur recovery rate, flowing out of the first-stage condenser (E-303), the process gas is heated and fed into the first and second catalytic conversion reactor (R-303/304) successively to produce sulfur. The gas from SRU is sent into the TGTU, which is mainly composed of hydrogenation reduction reaction and MDEA absorption processes. In the hydrogenation reduction reactor (R-401), most $\mathrm{SO}_{2}$ and sulfur are restored into $\mathrm{H}_{2} \mathrm{~S}$, which is then sent into the absorption column (Abs-402). Flowing out of the absorption column, the tail gas is fed into incinerator (F-402), where $\mathrm{H}_{2} \mathrm{~S}$ is converted into $\mathrm{SO}_{2}$. The high-temperature flue gas from incinerator is then discharged into the atmosphere through chimney. The heat of flue gas is recovered by the waste heat boiler. In the AWSU, the acid gas (main content $\mathrm{H}_{2} \mathrm{~S}$ ) is stripped from water through stripper column (Str-501).

\subsection{Operating conditions and specification}

The pressure, temperature and flow rate of feed gas are $8.30 \mathrm{MPa}, 35{ }^{\circ} \mathrm{C}$ and $1.25 \times 10^{5} \mathrm{Nm}^{3} / \mathrm{h}$, respectively. The composition of feed gas is: $\mathrm{CH}_{4} 76.52, \mathrm{C}_{2} \mathrm{H}_{6} 0.12, \mathrm{C}_{3} \mathrm{H}_{8}$ $0.01, \mathrm{H}_{2} \mathrm{~S}$ 14.14, $\mathrm{CO}_{2} 8.63, \mathrm{~N}_{2} 0.55, \mathrm{H}_{2} 0.02$ and $\mathrm{He} 0.01$ (mol\%). The fundamental operating data are listed in Table 1 . And the quality of treated gas should meet the specification as follows (Wang 2005): (1) $\mathrm{H}_{2} \mathrm{~S}: S_{1} \leq 6 \mathrm{mg} / \mathrm{Nm}^{3}$; (2) $\mathrm{CO}_{2}: S_{2} \leq 3 \mathrm{~V} \%$; (3) water dew point: $S_{3} \leq-15{ }^{\circ} \mathrm{C}$; (4) sulfur recovery rate: $S_{4} \geq 99.8 \%$; (5) $\mathrm{SO}_{2}: S_{5} \leq 960 \mathrm{mg} / \mathrm{Nm}^{3}$.

\subsection{Process simulation model}

The simulation model of whole high-sulfur natural gas purification process has been established by using software ProMax. The operating data in plant have been used to adjust ProMax process simulation model. Good agreements between operating data and simulation results are achieved and given in Table 2.

In Table 2, absolute deviation percent (AD \%) is used, defined by Eq. (1),

$\mathrm{AD} \%=100 \times\left|\frac{y_{i}-x_{i}}{y_{i}}\right|$

where $y_{\mathrm{i}}$ and $x_{\mathrm{i}}$ represent operating data and simulation data used to calculate $\mathrm{AD} \%$, respectively.

\section{Determination of decision variables for optimization model}

\subsection{Analysis of energy consumption distribution}

Comprehensive energy consumption distribution of each unit and the total was analyzed based on the plant data and is given in Table 3. Comprehensive energy consumption (CEC) is generally made up of fuel gas, electricity, 3.5 MPa medium-pressure steam (MPS), 0.4 MPa lowpressure steam (LPS) and water. The comprehensive 


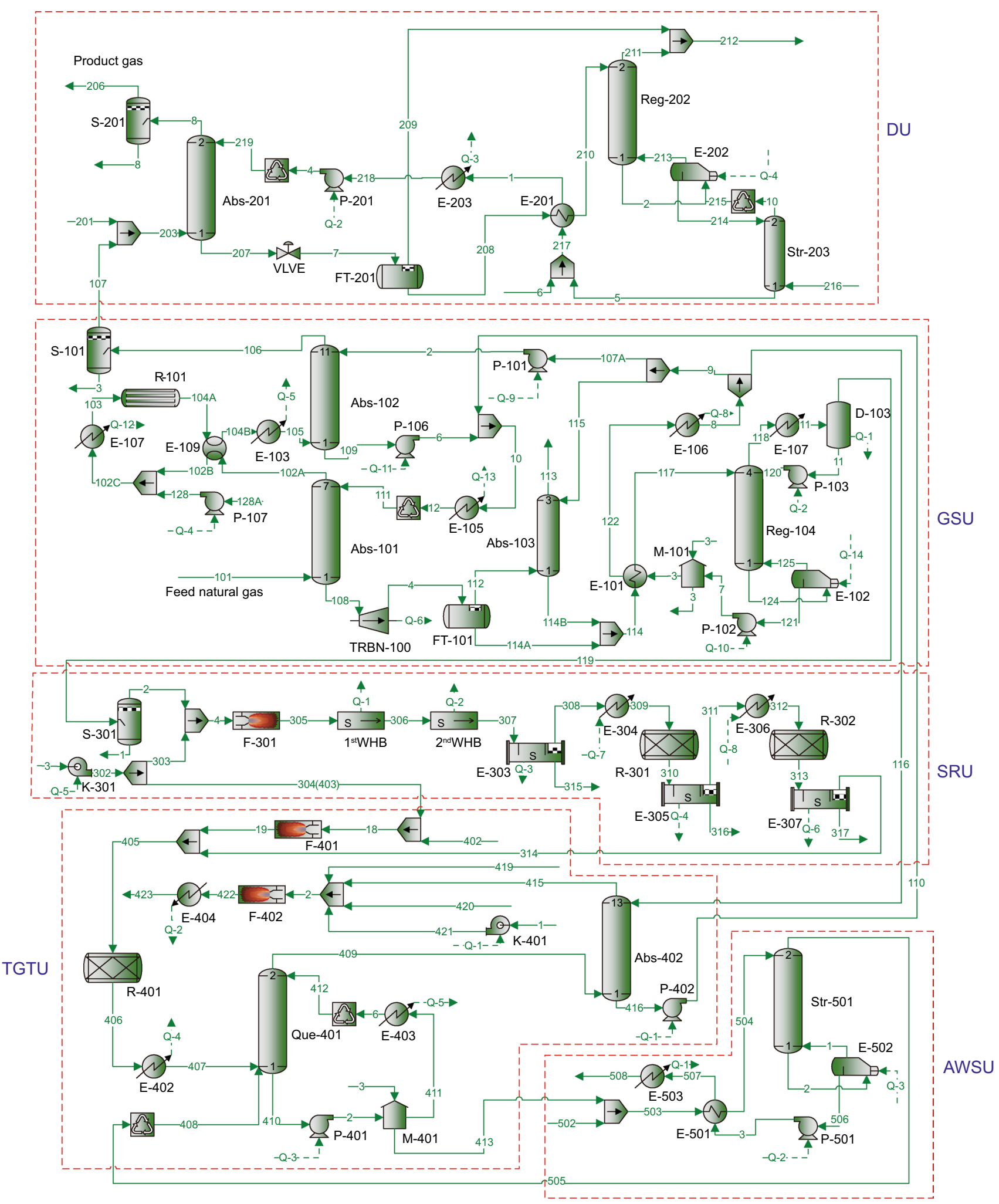

Fig. 2 The process flow diagram of gas purification plant 
Table 1 Operating data of the natural gas purification plant

\begin{tabular}{|c|c|c|c|c|c|}
\hline Unit & Items & Value & Unit & Items & Value \\
\hline \multirow[t]{4}{*}{ GSU } & MDEA temperature into the second absorption tower, ${ }^{\circ} \mathrm{C}$ & 39 & SRU & Claus furnace temperature, ${ }^{\circ} \mathrm{C}$ & 1070 \\
\hline & MDEA mass concentration, wt $\%$ & 50 & & Claus furnace air flow, $\mathrm{kNm}^{3} / \mathrm{h}$ & 38 \\
\hline & MDEA circulation rate, $\mathrm{t} / \mathrm{h}$ & 570 & & Tail gas $\mathrm{H}_{2} \mathrm{~S}_{2} \mathrm{SO}_{2}$ ratio & 4 \\
\hline & Regeneration tower reflux ratio, $\%$ & 0.89 & & $\mathrm{LP} / \mathrm{MP}$ pressure, $\mathrm{MPa} / \mathrm{MPa}$ & $0.45 / 3.5$ \\
\hline \multirow[t]{3}{*}{ DU } & TEG temperature into the absorption tower, ${ }^{\circ} \mathrm{C}$ & 55 & TGTU & Combustion air flow, $\mathrm{kNm}^{3} / \mathrm{h}$ & 16 \\
\hline & TEG mass concentration, $\%$ & 99.66 & & Quenched water circulation, $\mathrm{t} / \mathrm{h}$ & 524 \\
\hline & TEG circulation rate, $\mathrm{t} / \mathrm{h}$ & 3.8 & AWSU & Stripper column temperature, ${ }^{\circ} \mathrm{C}$ & 106 \\
\hline
\end{tabular}

Table 2 Comparison between simulation and plant data

\begin{tabular}{llll}
\hline Parameter, mol\% & Operating data & Simulation data & AD, $\%$ \\
\hline $\mathrm{H}_{2} \mathrm{~S}$ content of product gas & 0.00037 & 0.00038 & 2.70 \\
$\mathrm{H}_{2} \mathrm{O}$ content of product gas & 0.0036 & 0.0035 & 2.78 \\
$\mathrm{CO}_{2}$ content of product gas & 2.03 & 13.03 & 0 \\
$\mathrm{MDEA}$ content of lean amine & 13.125 & 12.818 & 0.008 \\
$\mathrm{MDEA}$ content of semi-lean amine & 12.819 & 0.29 & 0.008 \\
$\mathrm{SO}_{2}$ content of tail gas of Claus reaction & 0.33 & 0.00 & 7.50 \\
$\mathrm{H}_{2} \mathrm{~S}$ content of exhaust gas & 0.00 & 0.0205 & 0.00 \\
$\mathrm{SO}_{2}$ content of exhaust gas & 0.021 & & 2.50 \\
\hline
\end{tabular}

Table 3 Comprehensive energy consumption distribution

\begin{tabular}{|c|c|c|c|c|c|c|}
\hline Unit & $\mathrm{CEC}, \mathrm{MJ} / 10^{4} \mathrm{Nm}^{3}$ & $\begin{array}{l}\text { Fuel gas, } \mathrm{MJ} / 10^{4} \\
\mathrm{Nm}^{3}\end{array}$ & $\begin{array}{l}\text { Electricity, } \\
\mathrm{MJ} / 10^{4} \mathrm{Nm}^{3}\end{array}$ & MPS, MJ $/ 10^{4} \mathrm{Nm}^{3}$ & LPS, MJ $/ 10^{4} \mathrm{Nm}^{3}$ & $\begin{array}{l}\text { Water, } \\
\mathrm{MJ} / 10^{4} \\
\mathrm{Nm}^{3}\end{array}$ \\
\hline GSU & 17,136 & 0 & 737 & 917 & 14,861 & 621 \\
\hline DU & 172 & 83 & 11 & 78 & 0 & 0 \\
\hline SRU & $-16,025$ & 0 & 0 & $-13,413$ & -2936 & 324 \\
\hline TGTU & 5029 & 6864 & 262 & -408 & -2056 & 367 \\
\hline AWSU & 410 & 0 & 6 & 0 & 371 & 33 \\
\hline Total & 6723 & 6947 & 1016 & $-12,826$ & 10,240 & 1345 \\
\hline
\end{tabular}

energy consumption in the plant and in each unit can be calculated by Eq. (2), which is based on the Chinese industry standard 'Calculation method for energy consumption in petrochemical engineering design'(SH/T 3110-2001).

$E_{\mathrm{C}}=\left(c_{1} E_{\mathrm{E}}+c_{2} E_{\mathrm{FG}}+c_{3} E_{\mathrm{LPS}}+c_{4} E_{\mathrm{MPS}}+c_{5} E_{\mathrm{W}}\right) / m_{\mathrm{FNG}}$

In Eq. (2), $E_{\mathrm{C}}$ represents comprehensive energy consumption $\left(\mathrm{MJ} / 10^{4} \mathrm{Nm}^{3}\right) ; \mathrm{m}_{\mathrm{FNG}}$ is the flow rate of feed natural gas under the standard condition $\left(10^{4} \mathrm{Nm}^{3} / \mathrm{h}\right) ; E_{\mathrm{E}}$, $E_{\mathrm{FG}}, E_{\mathrm{LPS}}, E_{\mathrm{MPS}}$ and $E_{\mathrm{W}}$ represent the energy of electricity $(\mathrm{kW})$, fuel gas $(\mathrm{t} / \mathrm{h})$, low-pressure steam $(\mathrm{t} / \mathrm{h})$, mediumpressure steam $(\mathrm{t} / \mathrm{h})$ and water $(\mathrm{t} / \mathrm{h})$, respectively; $c_{1}-c_{5}$ represent corresponding equivalent coefficient of energy transfer medium.
The results show that GSU and TGTU are high comprehensive energy consumption units, and GSU takes about $75 \%$ of the TCEC in the four energy-consuming units. In GSU and TGTU, low-pressure steam for amine regeneration and fuel gas for hydrogenation reduction and tail gas combustion are main energy consumption, respectively. SRU is an exothermic unit with a large amount of $3.5 \mathrm{MPa}$ and $0.4 \mathrm{MPa}$ steam as by-product. DU and AWSU are low energy consumption units by contrast. So, the steam used by the purification process mainly relies on the steam derived from heat recovery of exothermic devices. And some pumps are also driven by the by-product steam. 


\subsection{Decision variables}

Based on the analysis of energy consumption distribution, to reduce complexity of optimization model, the operating parameters in DU and AWSU have not been considered as input variables in the optimization model. The operating parameters affecting energy consumption have been analyzed for GSU, SRU and TGTU through simulation by sensitivity analysis method (Hu 2013). Then, seven key parameters were selected as input variables of the optimization model given in Table 4 .

\section{Optimization model based on BP and NSGA-II}

\subsection{The objective function and constraints}

The objective functions of optimization are to minimize the TCEC and maximize the PGPR, which can be expressed mathematically as follows:

Minimize

$\operatorname{Object}(1)=E_{\mathrm{TCEC}}$

$\operatorname{Object}(2)=-m_{\mathrm{PNG}} / m_{\mathrm{FNG}}$

In Eq. (4), $m_{\mathrm{PNG}}$ and $m_{\mathrm{FNG}}$ represent the volume flow rate of purified natural gas and feed natural gas under the standard condition $\left(10^{4} \mathrm{Nm}^{3} / \mathrm{h}\right)$, respectively.

The quality specification $\left(S_{1}-S_{5}\right)$ that the treated gas should meet is regarded as the inequality constraint. The input variables are the seven key operating parameters $V_{1}-V_{7}$ in Table 4.

\subsection{Frame diagram of optimization process}

Figure 3 shows the frame diagram of the optimization process. Uniform design method (UD), which is a statistics experimental design method, similar to orthogonal design method, has been applied to design the data set for training

Table 4 Selected key operating parameter

\begin{tabular}{llc}
\hline Unit & Optimization variable & Range \\
\hline GSU & $\begin{array}{l}\text { The temperature of the amine solution into } \\
\text { the second absorption tower, } V_{1},{ }^{\circ} \mathrm{C}\end{array}$ & $35-45$ \\
& Amine solution circulation rate, $V_{2}, \mathrm{t} / \mathrm{h}$ & $540-600$ \\
& Amine solution concentration, $V_{3}, \mathrm{wt} \%$ & $45-54$ \\
& Regeneration tower reflux ratio, $V_{4}$ & $0.80-1$ \\
SRU & Tail gas $\mathrm{H}_{2} \mathrm{~S}_{\mathrm{SO}} \mathrm{S}_{2}$ ratio, $V_{5}$ & $2-6$ \\
TGTU & The combustion air flow, $V_{6}, \mathrm{kNm}^{3} / \mathrm{h}$ & $18-27$ \\
& Quenched water circulation, $V_{7}, \mathrm{t} / \mathrm{h}$ & $400-540$ \\
\hline
\end{tabular}

and testing the BP neural network model. Then, NSGA-II method has been used to optimize the seven input variables to obtain Pareto optimal set for minimum of TCEC and maximum of PGPR. The details about mentioned method and model will be presented in the following sections.

\section{Results and discussion}

\subsection{Design of training and testing data}

The UD method is based on the quasi-Monte Carlo method and proposed by Fang (1994). UD designs the experimental points to be scattered uniformly within the experimental domain. Compared with other conventional statistical experiment methods, UD is an effective approach and can further reduce the number of experimental trials which is proportional to the number of factor level (Fang and Ma 2001). For example, if a case with $\mathrm{m}$ factors (e.g., $x_{1}, x_{2}, x_{\mathrm{m}}$ ) and $s$ levels for each factor, the comprehensive needs $\mathrm{m}^{\mathrm{s}}$ experiments and orthogonal design method needs $s^{2}$ experiments, while UD only needs number of s experiments. The UD tables for designing different experiment trials have been introduced in the literature (Fang, 1994).

The table $\mathrm{U}_{21}\left(10^{7}\right)$ of UD experiments has been applied to arrange the investigated seven operating parameters as listed in Table 5. The process parameters have been graded into 20 even levels, and 21 experimental trials have been determined. For each input parameter, in the range of the minimum and the maximum, there exists an arithmetic progression with 21 data which are numbered from No. 1 to No. 21 in order. For each experimental trial, according to the uniform experiment table, it is a combination of seven input parameters.

To further evaluate reliability of the BP model, different sample sizes such as 50,100, 300 and 600 have been used. For all the sample sizes, $90 \%$ data have been used to train the model, while the left, i.e., $10 \%$, to test the accuracy of the model.

To quantify the difference between predictions of BP model and ProMax results, average relative deviation percent (AAD \%) is defined by the following equation

$\mathrm{AAD} \%=\frac{100}{n} \sum_{i=1}^{n}\left|\frac{y_{i}-x_{i}}{y_{i}}\right|$

where $y_{\mathrm{i}}, x_{\mathrm{i}}$ and $n$ represent ProMax results, BP model predictions and number of data point used to calculate AAD $\%$, respectively. AAD $\%$ for sample sizes, i.e., $21,100,400$ and 600 , was $1.11 \%, 0.69 \%, 0.62 \%$ and $0.61 \%$, respectively. Although the accuracy of the sample size with 21 was lower than the other, it is still acceptable for engineering application. To present this method much clearly, we decide to keep 


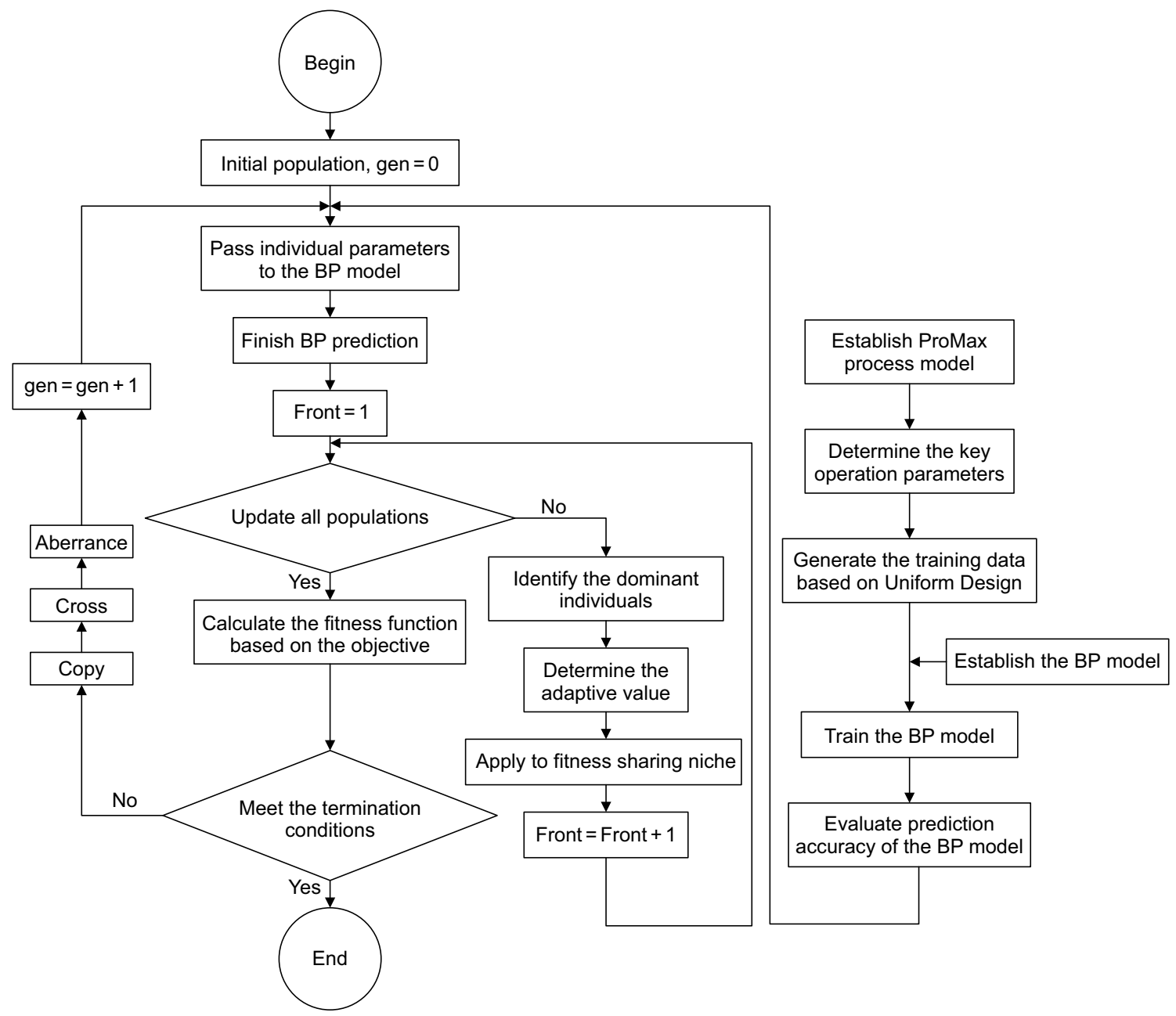

Fig. 3 Frame diagram of NSGA-II optimization model

the sample size with 21 in paper to show the whole process that how to set up the UD experiment table and show the input and output parameters of BP model in details.

In Table 5, a base experiment point, composed with the actual operating parameters, was added. Among the 22 experiment points, the 7th, 14th, 21st and the Base were chosen as testing series to evaluate the accuracy of BP model, and the others were selected as training series. All 22 operating conditions have been calculated by the gas purification process simulation model. Then, the simulation results of the quality specification $\left(S_{1}-S_{5}\right)$, TCEC and PGPR are given in Table 6. Among these experiment points, No. 9 attracts much attention for its lowest TCEC, which reduced by $13.5 \%$ compared with the Base. This also reflects that the UD method can efficiently design and get the representative experiment trails.

\subsection{Prediction of BP neural network model}

There are multitudes of different types of artificial neural networks. The multilayer perceptron with the back-propagation of error algorithm is more popular and is used in this paper.

The MATLAB neural network toolbox was used to establish the BP neural network. The main function was set as 'net $=$ newff $(\operatorname{minmax}(\mathrm{P}),[15,7],\{$ 'tansig', 'logsig' $\}$, 'traingdx')'. The input data were normalized to $[-1,+1]$ by the 'minmax' function before training.

There are three layers (input, hidden and output layers) in the training algorithms. After a large amount of practice and comparison for different numbers of neurons, 15 neurons for the hidden layer were recommended. The transfer functions 'tansig' for input-hidden layer 
Table 5 Training and testing data for BP model

\begin{tabular}{|c|c|c|c|c|c|c|c|}
\hline \multirow[t]{2}{*}{ No. } & \multicolumn{7}{|c|}{ Input variable } \\
\hline & $\overline{V_{1},{ }^{\circ} \mathrm{C}}$ & $V_{2}, \mathrm{t} / \mathrm{h}$ & $V_{3}$, wt $\%$ & $V_{4}$ & $V_{5}$ & $V_{6}, \mathrm{kNm}^{3} / \mathrm{h}$ & $V_{7}, \mathrm{t} / \mathrm{h}$ \\
\hline 1 & 35.0 & 552 & 47.70 & 0.88 & 4.40 & 25.20 & 526 \\
\hline 2 & 35.5 & 567 & 50.85 & 0.97 & 2.60 & 22.95 & 505 \\
\hline 3 & 36.0 & 582 & 54.00 & 0.84 & 5.20 & 20.70 & 484 \\
\hline 4 & 36.5 & 597 & 47.25 & 0.93 & 3.40 & 18.45 & 463 \\
\hline 5 & 37.0 & 546 & 50.40 & 0.80 & 6.00 & 26.10 & 442 \\
\hline 6 & 37.5 & 561 & 53.55 & 0.89 & 4.20 & 23.85 & 421 \\
\hline 7 & 38.0 & 576 & 46.80 & 0.98 & 2.40 & 21.60 & 400 \\
\hline 8 & 38.5 & 591 & 49.95 & 0.85 & 5.00 & 19.35 & 533 \\
\hline 9 & 39.0 & 540 & 53.10 & 0.94 & 3.20 & 27.00 & 512 \\
\hline 10 & 39.5 & 555 & 46.35 & 0.81 & 5.80 & 24.75 & 491 \\
\hline 11 & 40.0 & 570 & 49.50 & 0.90 & 4.00 & 22.50 & 470 \\
\hline 12 & 40.5 & 585 & 52.65 & 0.99 & 2.20 & 20.25 & 449 \\
\hline 13 & 41.0 & 600 & 45.90 & 0.86 & 4.80 & 18.00 & 484 \\
\hline 14 & 41.5 & 549 & 49.05 & 0.95 & 3.00 & 25.65 & 407 \\
\hline 15 & 42.0 & 564 & 52.20 & 0.82 & 5.60 & 23.40 & 540 \\
\hline 16 & 42.5 & 579 & 45.45 & 0.91 & 3.80 & 21.15 & 519 \\
\hline 17 & 43.0 & 594 & 48.60 & 1.00 & 2.00 & 18.90 & 498 \\
\hline 18 & 43.5 & 543 & 51.75 & 0.87 & 4.60 & 26.55 & 477 \\
\hline 19 & 44.0 & 558 & 45.00 & 0.96 & 2.80 & 24.30 & 456 \\
\hline 20 & 44.5 & 573 & 48.15 & 0.83 & 5.40 & 22.05 & 435 \\
\hline 21 & 45.0 & 588 & 51.30 & 0.92 & 3.60 & 19.80 & 414 \\
\hline Base & 39.0 & 570 & 49.60 & 0.89 & 4.00 & 23.00 & 524 \\
\hline
\end{tabular}

Opt. represents the selected Pareto optimal point

and 'logsig' for hidden-output layer were applied. The 'traingdx' was applied as training function. The 'sim(net, $P \_$test)' was constructed as the prediction function. The input variables of the BP neural network were the seven operating parameters in Table 5, while the seven output variables were the quality specification $\left(S_{1}-S_{5}\right)$, TCEC and PGPR in Table 6.

Table 7 shows the agreement between predictive values by BP neural network model and the simulation values by ProMax model. It can be seen that the accuracy of BP prediction is high, which will provide a reliable basis for the further optimization.

\subsection{Pareto optimal solutions}

The optimization goal is to minimize TCEC and maximize the PGPR. Since two objective functions cannot be optimized simultaneously, the non-dominated sorting genetic algorithm (NSGA-II) has been applied to achieve the Pareto optimal solutions. The real-coded NSGA-II has been implemented in MATLAB. In the NSGA-II model, the values of population size, maximum generations and tournament pool size are 100, 300 and 2, respectively; replace proportion, crossover probability and mutation probability are $0.9,0.9$ and 0.14 , respectively; the crossover method and mutation method are two points and selective.

The Pareto optimal solutions have good convergence and uniformity indicated in Fig. 4. It can be concluded that no objective function for a Pareto solution can be improved without worsening the other objective function. All these points are optimal and no superior to others. In Fig. 4, among the Pareto front curve, three points A, B and $\mathrm{C}$ have been chosen to be further discussed. Point $\mathrm{A}$ has the lowest TCEC with the lowest PGPR; point B has the medium PGPR with medium TCEC; point $\mathrm{C}$ has the highest PGPR with medium TCEC. The process optimization should consider the control mode and accuracy of the operating parameters in the gas processing field. So the diversity of Pareto optimal solutions can supply more possibility for the gas processing field to select based on its actual condition.

The values of input variables, TCEC and PGPR for the three points are listed in Table 8. TCEC of all the points is lower than the Base about $13.4 \%$, and PGPR is higher than the Base about $0.2 \%$. As it is known that the decrease in the values of $V_{1}, V_{2}, V_{5}, V_{6}$ and $V_{7}$ can 
Table 6 Training and testing data for BP model

\begin{tabular}{|c|c|c|c|c|c|c|c|}
\hline \multirow[t]{2}{*}{ No. } & \multicolumn{7}{|c|}{ Output parameter } \\
\hline & $S_{1}, \mathrm{mg} / \mathrm{Nm}^{3}$ & $S_{2}, \mathrm{~V} \%$ & $S_{3},{ }^{\circ} \mathrm{C}$ & $S_{4}, \%$ & $S_{5}, \mathrm{mg} / \mathrm{Nm}^{3}$ & TCEC, $\mathrm{MJ} / 10^{4} \mathrm{Nm}^{3}$ & PGPR, $\%$ \\
\hline 1 & 5.82 & 2.02 & -20.45 & 99.883 & 759 & 6206 & 78.097 \\
\hline 2 & 5.97 & 2.06 & -20.07 & 99.882 & 787 & 6044 & 78.154 \\
\hline 3 & 5.87 & 2.09 & -19.73 & 99.886 & 785 & 6497 & 78.147 \\
\hline 4 & 5.89 & 2.00 & -19.06 & 99.877 & 865 & 7177 & 78.024 \\
\hline 5 & 5.83 & 2.06 & -18.49 & 99.881 & 750 & 6259 & 78.178 \\
\hline 6 & 5.97 & 2.10 & -18.14 & 99.884 & 760 & 6262 & 78.185 \\
\hline 7 & 5.89 & 1.98 & -17.29 & 99.875 & 836 & 6941 & 78.021 \\
\hline 8 & 5.89 & 2.04 & -16.93 & 99.878 & 838 & 7193 & 78.089 \\
\hline 9 & 5.82 & 2.10 & -16.40 & 99.885 & 845 & 5818 & 78.215 \\
\hline 10 & 5.93 & 1.99 & -15.86 & 99.869 & 824 & 6915 & 78.062 \\
\hline 11 & 5.92 & 2.02 & -15.50 & 99.875 & 819 & 6948 & 78.073 \\
\hline 12 & 5.90 & 2.06 & -15.09 & 99.881 & 812 & 7008 & 78.076 \\
\hline 13 & 5.71 & 1.94 & -16.45 & 99.871 & 886 & 8092 & 77.945 \\
\hline 14 & 5.99 & 2.02 & -15.85 & 99.873 & 797 & 6756 & 78.119 \\
\hline 15 & 5.97 & 2.06 & -15.44 & 99.876 & 800 & 6981 & 78.124 \\
\hline 16 & 5.97 & 1.95 & -15.22 & 99.864 & 891 & 7655 & 77.983 \\
\hline 17 & 5.89 & 1.98 & -15.10 & 99.876 & 858 & 7720 & 77.980 \\
\hline 18 & 5.87 & 2.06 & -15.49 & 99.875 & 766 & 6879 & 78.167 \\
\hline 19 & 5.98 & 1.95 & -15.43 & 99.861 & 869 & 7504 & 78.022 \\
\hline 20 & 5.99 & 1.98 & -15.31 & 99.863 & 881 & 7737 & 78.024 \\
\hline 21 & 5.96 & 2.01 & -15.14 & 99.871 & 861 & 7804 & 78.025 \\
\hline Base & 5.84 & 2.03 & -16.42 & 99.879 & 876 & 6722 & 78.081 \\
\hline
\end{tabular}

Table 7 Comparison between BP prediction and ProMax simulation

\begin{tabular}{|c|c|c|c|c|c|c|}
\hline \multirow[t]{2}{*}{ Parameter } & \multicolumn{3}{|l|}{ No. 7} & \multicolumn{3}{|l|}{ No. 14} \\
\hline & Simulation & Prediction & $\mathrm{AD}, \%$ & Simulation & Prediction & $\mathrm{AD}, \%$ \\
\hline$S_{1}$ & 5.89 & 5.85 & 0.533 & 5.99 & 5.79 & 3.33 \\
\hline$S_{2}$ & 1.98 & 1.96 & 1.20 & 2.02 & 2.01 & 0.363 \\
\hline$S_{3}$ & -17.29 & -17.75 & 2.66 & -15.85 & -15.74 & 0.695 \\
\hline$S_{4}$ & 99.875 & 99.869 & 0.006 & 99.873 & 99.875 & 0.002 \\
\hline$S_{5}$ & 836 & 819 & 2.04 & 797 & 785 & 1.52 \\
\hline Obj. 1 & 6941 & 6961 & 0.289 & 6756 & 6744 & 0.183 \\
\hline \multirow[t]{2}{*}{ Obj. 2} & 78.02 & 78.00 & 0.027 & 78.11 & 78.07 & 0.051 \\
\hline & \multicolumn{3}{|l|}{ No. 21} & \multicolumn{3}{|l|}{ Base } \\
\hline$S_{1}$ & 5.96 & 5.99 & 0.461 & 5.84 & 5.98 & 2.38 \\
\hline$S_{2}$ & 2.01 & 1.99 & 0.889 & 2.03 & 2.03 & 0.110 \\
\hline$S_{3}$ & -15.14 & -15.12 & 0.137 & -16.42 & -16.01 & 2.48 \\
\hline$S_{4}$ & 99.871 & 99.875 & 0.004 & 99.879 & 99.874 & 0.006 \\
\hline$S_{5}$ & 861 & 878 & 1.93 & 876 & 840 & 4.13 \\
\hline Obj. 1 & 7804 & 7502 & 3.87 & 6723 & 6646 & 1.14 \\
\hline Obj. 2 & 78.02 & 77.98 & 0.055 & 78.08 & 78.10 & 0.027 \\
\hline
\end{tabular}

Obj. 1 and Obj. 2 represent the objective functions of TCEC and PGPR, respectively 


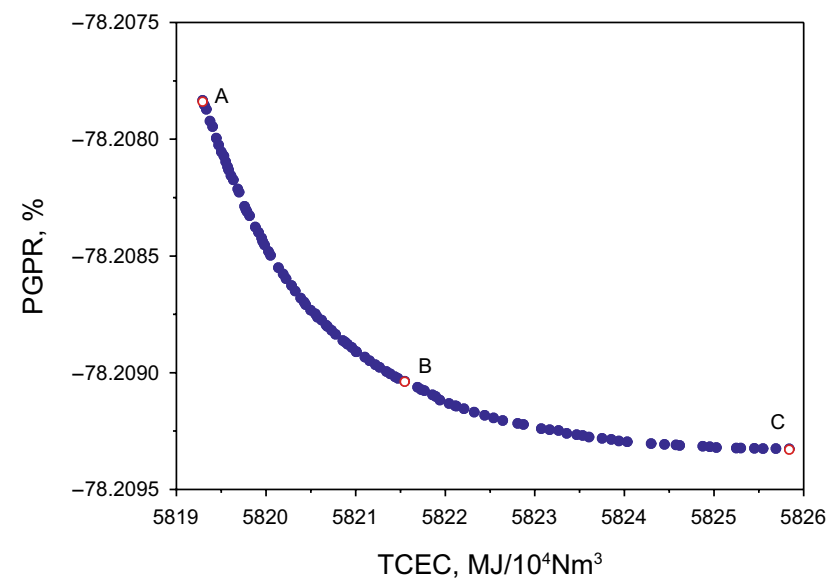

Fig. 4 Pareto front of two objectives TCEC and PGPR

decrease TCEC, while the increase in the values of $V_{3}$, $V_{4}, V_{5}$ and $V_{7}$ can increase PGPR. Compared with the Base, all the points $A, B, C$ have lower the amine solution temperature into the second absorption tower $V_{1}$, the amine solution circulation rate $V_{2}$, the tail gas $\mathrm{H}_{2} \mathrm{~S} /$ $\mathrm{SO}_{2}$ ratio $V_{5}$, and the quenched water circulation $V_{7}$, while they have higher the amine solution concentration $V_{3}$, the regeneration tower reflux ratio $V_{4}$ and the combustion air flow $V_{6}$. The adjustment results of these operating parameter $V_{1}-V_{7}$, can offer reference for the optimal operation in gas processing field. Furthermore, the objective functions have been recalculated by using the ProMax process simulation model in the same operating conditions and are given in Table 8 .

\section{Conclusions}

The optimization of a whole high-sulfur natural gas purification plant was investigated to reduce the TCEC and further improve the PGPR. To provide sufficient data and determine key operating parameters, a steady-state simulation model of high-sulfur natural gas purification process including GSU, DU, SRU, TGTU and AWSU was set up by using software ProMax. And the model has good agreement compared with the actual operating data. Seven key operating parameters, as input variables in optimization model, were determined based on the analysis of comprehensive energy consumption distribution. To solve the problem that the process model did not converge in some conditions for the existence of nested loops in it, BP neural network was applied to substitute the simulation model to predict the relative parameters needed in the optimization model. The UD method and the table $\mathrm{U}_{21}\left(10^{7}\right)$ were applied to design the experiment points for training and testing BP model. The BP model can offer high prediction accuracy compared with the simulation model. NSGA-II was established to optimize the two objectives, and 100 Pareto optimal solutions were achieved. Three optimal points $\mathrm{A}, \mathrm{B}$ and $\mathrm{C}$ were selected and evaluated further. The TCEC was reduced by $13.4 \%$ and the PGPR was improved by $0.2 \%$ compared with the Base operation condition.

Further investigation will be carried out to verify the stability and reliability by using much more plant operating data. It demonstrates the applicability and feasibility of BP neural network and NSGA-II to resolve global optimization problem in the field of the high-sulfur gas purification plant.

Table 8 Selected Pareto optimization results for TCEC and PGPR

\begin{tabular}{|c|c|c|c|c|c|c|c|}
\hline No. & $V_{1},{ }^{\circ} \mathrm{C}$ & $V_{2}, \mathrm{t} / \mathrm{h}$ & $V_{3}, \mathrm{wt} \%$ & $V_{4}$ & $V_{5}$ & $V_{6}, \mathrm{kNm}^{3} / \mathrm{h}$ & $V_{7}, \mathrm{t} / \mathrm{h}$ \\
\hline$A$ & 35.0 & 540 & 54.00 & 1.00 & 2.35 & 27.00 & 475 \\
\hline$B$ & 36.2 & 553 & 54.00 & 1.00 & 2.32 & 27.00 & 474 \\
\hline$C$ & 37.9 & 562 & 54.00 & 1.00 & 2.51 & 27.00 & 476 \\
\hline \multirow[t]{2}{*}{ No. } & & \multicolumn{3}{|l|}{ NSGA-II } & \multicolumn{3}{|c|}{ ProMax } \\
\hline & & TCEC, $\mathrm{MJ} / 10^{4} \mathrm{Nm}^{3}$ & \multicolumn{2}{|c|}{ PGPR, $\%$} & \multicolumn{2}{|c|}{ TCEC, $\mathrm{MJ} / 10^{4} \mathrm{Nm}^{3}$} & PGPR, $\%$ \\
\hline$A$ & & 5819 & \multicolumn{2}{|c|}{78.2078} & \multicolumn{2}{|c|}{5699} & 78.2391 \\
\hline$B$ & & 5822 & \multicolumn{2}{|c|}{78.2090} & \multicolumn{2}{|c|}{5721} & 78.2421 \\
\hline$C$ & & 5826 & \multicolumn{2}{|c|}{78.2093} & \multicolumn{2}{|c|}{5780} & 78.2469 \\
\hline
\end{tabular}


Acknowledgements Financial support from National Science and Technology Major Project of the Ministry of Science and Technology of China (Grant No. 2016ZX05017-004) is gratefully acknowledged.

Open Access This article is distributed under the terms of the Creative Commons Attribution 4.0 International License (http://creativeco mmons.org/licenses/by/4.0/), which permits unrestricted use, distribution, and reproduction in any medium, provided you give appropriate credit to the original author(s) and the source, provide a link to the Creative Commons license, and indicate if changes were made.

\section{References}

Abdulrahman RK, Sebastine IM. Natural gas sweetening process simulation and optimization: a case study of Khurmala field in Iraqi Kurdistan region. J Nat Gas Sci Eng. 2013;14:116-20. https://doi. org/10.1016/j.jngse.2013.06.005.

Adewale R, Salem DJ, Berrouk AS, Dara S. Simulation of hydrogen production from thermal decomposition of hydrogen sulfide in sulfur recovery units. J Clean Prod. 2016;112:4815-25. https:// doi.org/10.1016/j.jclepro.2015.08.021.

Ahmadi MA, Soleimani R, Bahadori A. A computational intelligence scheme for prediction equilibrium water dew point of natural gas in TEG dehydration systems. Fuel. 2014;137:145-54. https ://doi.org/10.1016/j.fuel.2014.07.072.

Al-Lagtah NMA, Al-Habsi S, Onaizi SA. Optimization and performance improvement of Lekhwair natural gas sweetening plant using Aspen HYSYS. J Nat Gas Sci Eng. 2015;26:367-81. https ://doi.org/10.1016/j.jngse.2015.06.030.

Azizkhani JS, Jazayeri-Rad H, Nabhani N. Design of an ensemble neural network to improve the identification performance of a gas sweetening plant using the negative correlation learning and genetic algorithm. J Nat Gas Sci Eng. 2014;21:26-39. https:// doi.org/10.1016/j.jngse.2014.07.012.

Behroozsarand A, Zamaniyan A. Multi-objective optimization scheme for industrial synthesis gas sweetening plant in GTL process. J Nat Gas Chem. 2011;20:99-109. https://doi. org/10.1016/S1003-9953(10)60153-3.

Boyaghchi FA, Chavoshi M. Multi-criteria optimization of a micro solar-geothermal CCHP system applying water/CuO nanofluid based on exergy, exergoeconomic and exergoenvironmental concepts. Appl Therm Eng. 2017;112:660-75. https://doi. org/10.1016/j.applthermaleng.2016.10.139.

Cui YF, Geng ZQ, Zhu QX, Han Y. Review: multi-objective optimization methods and application in energy saving. Energy. 2017;125:681-704. https://doi.org/10.1016/j.energ y.2017.02.174.

Dai XH, Li MQ, Kou JS. Survey on the theory of genetic algorithms. Control Decis. 2000;15(3):263-8 (in Chinese).

Damavandi MD, Forouzanmehr M, Safikhani H. Modeling and Pareto based multi-objective optimization of wavy fin-and-elliptical tube heat exchangers using CFD and NSGA-II algorithm. Appl Therm Eng. 2017;111:325-39. https://doi.org/10.1016/j. applthermaleng.2016.09.120.

Darwish NA, Hilal N. Sensitivity analysis and faults diagnosis using artificial neural networks in natural gas TEG-dehydration plants. Chem Eng J. 2008;137:189-97. https://doi.org/10.1016/j. cej.2007.04.008.

Deb K, Agrawal S, Pratap A, Meyarivan T. A fast elitist nondominated sorting genetic algorithm for multi-objective optimization: NSGA_II. In: Sixth international conference on parallel problem solving from nature 2000. p. 849-58. https:// doi.org/10.1007/3-540-45356-3 83.

Deng QH, Wang D, Zhao H, Huang WT, Shao S, Feng ZP. Study on performances of supercritical $\mathrm{CO}_{2}$ recompression Brayton cycles with multi-objective optimization. Appl Therm Eng. 2017;114:1335-42. https://doi.org/10.1016/j.appltherma leng.2016.11.055.

Fang KT. Uniform design and uniform design tables. Beijing: Science Press; 1994 (in Chinese).

Fang KT, Ma CX. Orthogonal and uniform experimental design. Beijing: Science Press; 2001 (in Chinese).

Foo DCY, Ooi REH, Tan RR, Lee JY. Process integration approaches to optimal planning of unconventional gas field development. Chem Eng Sci. 2016;150:85-93. https://doi.org/10.1016//j. ces.2016.04.049.

Ghiasi MM, Bahadori A, Zendehboudi S. Estimation of triethylene glycol (TEG) purity in natural gas dehydration units using fuzzy neural network. J Nat Gas Sci Eng. 2014;17:26-32. https://doi. org/10.1016/j.jngse.2013.12.008.

Guo QL. Evaluation and decision analysis of oil and gas exploration targets. Beijing: Petroleum Industry Press; 2004 (in Chinese).

$\mathrm{He}$ C, You FQ. Towards more cost-effective and greener chemicals production from shale gas by integrating with bioethanol dehydration: novel process design and optimization. AIChE J. 2015;61(4):1184-208. https://doi.org/10.1002/aic.14713.

$\mathrm{Hu}$ SP. Process modeling and parameter optimization of high-sulfur natural gas purification plant. Beijing: China University of Petroleum; 2013 (in Chinese).

Jassim MS. Sensitivity analyses and optimization of a gas sweetening plant for hydrogen sulfide and carbon dioxide capture using methyldiethanolamine solutions. J Nat Gas Sci Eng. 2016;36:17583. https://doi.org/10.1016/j.jngse.2016.10.012.

Jia QF, Liu HJ, Zhang L, Tong LZ, et al. Study of process optimization on Qiudong 1\# gas treatment unit. Chem Eng Oil Gas. 2009;38(5):386-9 (in Chinese).

Li Q. Energy analysis and optimization for high sour natural gas purification plant. Beijing: China University of Petroleum; 2012 (in Chinese).

Li YB, Li XF, Yao YD. Variation laws of $\mathrm{H}_{2} \mathrm{~S}$ concentration in the development process of sour gas reservoir. Acta Petrolei Sinica. 2007;28(6):99-102 (in Chinese).

Long ZB, Liu J, Wu X. Process schemes selection and simulation evaluation of high sour natural gas desulphurization. Technol Dev Chem Ind. 2007:36(12):28-32 (in Chinese).

Louis G, Maxime TG, Francois MP. Review of utilization of genetic algorithms in heat transfer problems. Int J Heat Mass Transf. 2009;52(4):2169-88. https://doi.org/10.1016/j.ijheatmasstrans fer.2008.11.015.

Manenti F, Papasidero D, Bozzano G, Ranzi E. Model-based optimization of sulfur recovery units. Comput Chem Eng. 2014;66:244-51. https://doi.org/10.1016/j.compchemeng.2014.01.019.

National Energy Administration, Development Research Center of the State Council and Ministry of Natural Resources of the People's Republic of China. In: China natural gas development report. Beijing: Petroleum Industry Press; 2016 (in Chinese).

Neri F, Carlos C. Memetic algorithms and memetic computing optimization: a literature review. Swarm Evol Comput. 2012;2:1-14. https://doi.org/10.1016/j.swevo.2011.11.003.

Peter L, Hussain A, Follmann M, Melin T, Hägg MB. $\mathrm{CO}_{2}$ removal from natural gas by employing amine absorption and membrane technology - a technical and economical analysis. Chem Eng J. 2011;172:952-60. https://doi.org/10.1016/j.cej.2011.07.007.

Qiu K, Shang JF, Ozturk M, et al. Studies of methyldiethanolamine process simulation and parameters optimization for high-sulfur gas sweetening. J Nat Gas Sci Eng. 2014;21:379-85. https://doi. org/10.1016/j.jngse.2014.08.023. 
Rouzbahani AN, Bahmani M, Shariati J, Tohidian T, Rahimpour MR. Simulation, optimization, and sensitivity analysis of a natural gas dehydration unit. J Nat Gas Sci Eng. 2014;21:159-69. https://doi. org/10.1016/j.jngse.2014.07.025.

Salooki MK, Abedini R, Adib H, Koolivand H. Design of neural network for manipulating gas refinery sweetening regenerator column outputs. Sep Purif Technol. 2011;82:1-9. https://doi. org/10.1016/j.seppur.2011.07.015.

Santos MGRS, Correia LMS, de Medeiros JL, de Queiroz FAO. Natural gas dehydration by molecular sieve in offshore plants: impact of increasing carbon dioxide content. Energy Convers Manag. 2017;149:760-73. https://doi.org/10.1016/j.encon man.2017.03.005.

Singh K, Das R. An experimental and multi-objective optimization study of a forced draft cooling tower with different fills. Energy Convers Manag. 2016;111:417-30. https://doi.org/10.1016/j. enconman.2015.12.080
Srinivas N, Deb K. Multi-objective optimization using non-dominated sorting in genetic algorithms. Evol Comput. 1994;2:221-48. https ://doi.org/10.1162/evco.1994.2.3.221.

Wahedi YA, Torres AI, Hashimi SA, Dowling NI, Daoutidis P, Tsapatsis $\mathrm{M}$. Economic assessment of temperature swing adsorption systems as claus tail gas clean up units. Chem Eng Sci. 2015;126:186-95. https://doi.org/10.1016/j.ces.2014.12.015.

Wang KY. Natural gas purification process-desulfurization, decarbonization, dehydration, sulfur recovery and the tail gas treatment. Beijing: Petroleum Industry Press; 2005 (in Chinese).

Yu HM, Yao PJ, Yuan Y. Improved genetic algorithm/simulated annealing for large system energy integration. J Chem Ind Eng. 1998;49(6):655-61 (in Chinese).

Zarei S, Ganji H, Sadi M, Rashidzadeh M. Thermo-kinetic modeling and optimization of the sulfur recovery unit thermal stage. Appl Therm Eng. 2016;103:1095-104. https://doi.org/10.1016/j.applt hermaleng.2016.05.012. 